\title{
A LAGRANGIAN APPROACH FOR THE INCOMPRESSIBLE NAVIER-STOKES EQUATIONS WITH VARIABLE DENSITY
}

\author{
RAPHAËL DANCHIN AND PIOTR BOGUSEAW MUCHA
}

\begin{abstract}
Here we investigate the Cauchy problem for the inhomogeneous Navier-Stokes equations in the whole $n$-dimensional space. Under some smallness assumption on the data, we show the existence of global-in-time unique solutions in a critical functional framework. The initial density is required to belong to the multiplier space of $\dot{B}_{p, 1}^{n / p-1}\left(\mathbb{R}^{n}\right)$. In particular, piecewise constant initial densities are admissible data provided the jump at the interface is small enough, and generate global unique solutions with piecewise constant densities. Using Lagrangian coordinates is the key to our results as it enables us to solve the system by means of the basic contraction mapping theorem. As a consequence, conditions for uniqueness are the same as for existence.
\end{abstract}

MSC: 35Q30, 76D05

Key words: Inhomogeneous Navier-Stokes equations, critical regularity, piecewise constant density, Besov spaces, Lagrangian coordinates.

\section{INTRODUCTION}

We address the well-posedness issue for the the incompressible Navier-Stokes equations with variable density in the whole space $\mathbb{R}^{n}$ :

$$
\left\{\begin{array}{l}
\partial_{t} \rho+u \cdot \nabla \rho=0, \\
\rho\left(\partial_{t} u+u \cdot \nabla u\right)-\mu \Delta u+\nabla P=0, \\
\operatorname{div} u=0, \\
\left.u\right|_{\mid t=0}=u_{0} .
\end{array}\right.
$$

Above $\rho=\rho(t, x) \in \mathbb{R}_{+}$stands for the density, $u=u(t, x) \in \mathbb{R}^{N}$, for the velocity field and $P=P(t, x) \in \mathbb{R}$, for the pressure field. The viscosity coefficient $\mu$ is a given positive real number. We supplement this system with the following boundary conditions:

- the velocity $u$ tends to 0 at infinity,

- the density tends to some positive constant $\rho^{*}$ at infinity.

The exact meaning of those boundary conditions will be given by the functional framework in which we shall solve the system. In what follows, we will take $\rho^{*}=1$ to simplify the presentation.

This old system has known a renewed interest recently, among the mathematics community. The existence of strong smooth solutions with positive density has been established in e.g. [11] whereas the theory of global weak solutions with finite energy has been performed in the book [12] by P.-L. Lions (see also the references therein, and the monograph [3]). As pointed out in [5], it is possible to construct strong unique solutions for some classes of smooth enough data with vanishing density.

In the present paper, we aim at solving the above system in critical functional spaces, that is in spaces which have the same invariance with respect to time and space dilation as the system itself (see e.g. [6] or [7] for more explanations about this nowadays classical approach). In this

Date: September 4, 2016. 
framework, it has been stated in $[1,6]$ that, for data $\left(\rho_{0}, u_{0}\right)$ such that

$$
\left(\rho_{0}-1\right) \in \dot{B}_{p, 1}^{n / p}\left(\mathbb{R}^{n}\right), \quad u_{0} \in \dot{B}_{p, 1}^{n / p-1}\left(\mathbb{R}^{n}\right) \quad \text { with } \quad \operatorname{div} u_{0}=0
$$

and that, for a small enough constant $c$,

$$
\left\|\rho_{0}-1\right\|_{\dot{B}_{p, 1}^{n / p}\left(\mathbb{R}^{n}\right)}+\mu^{-1}\left\|u_{0}\right\|_{\dot{B}_{p, 1}^{n / p-1}} \leq c,
$$

we have for any $p \in[1,2 n)$ :

- existence of a global solution $(\rho, u, \nabla P)$ with $\rho-1 \in \mathcal{C}_{b}\left(\mathbb{R}_{+} ; \dot{B}_{p, 1}^{n / p}\right), u \in \mathcal{C}_{b}\left(\mathbb{R}_{+} ; \dot{B}_{p, 1}^{n / p-1}\right)$ and $\partial_{t} u, \nabla^{2} u, \nabla P \in L_{1}\left(\mathbb{R}_{+} ; \dot{B}_{p, 1}^{n / p-1}\right)$;

- uniqueness in the above space if in addition $p \leq n$.

Those results have been somewhat extended in [2] where it has been noticed that $\rho_{0}-1$ may be taken in a larger Besov space, with another Lebesgue exponent.

The above results are based on maximal regularity estimates in Besov spaces for the evolutionary Stokes system, and on the Schauder-Tychonoff fixed point theorem. In effect, owing to the hyperbolicity of the density equation, there is a loss of one derivative in the stability estimates thus precluding the use of the contraction mapping (or Banach fixed point) theorem. As a consequence, the conditions for uniqueness are stronger than those for existence.

Let us also point out that all the above results concerning existence with uniqueness require the density to be at least uniformly continuous. This condition has been somewhat weakened recently by P. Germain in [9]. However, there, initial densities with jump across an interface cannot be considered.

In the present paper, we aim at solving System (0.1) in the Lagrangian coordinates. The main motivation is that the density is constant along the flow so that only the (parabolic type) equation for the velocity has to be considered. We shall show that, after performing this change of coordinates, solving (0.1) may be done by means of the Banach fixed point theorem. As a consequence, the condition for uniqueness need not be stronger than that for the existence, and the flow map is Lipschitz continuous.

Our main result states the global in time existence of regular solutions to the inhomogeneous Navier-Stokes equations in $\mathbb{R}^{n}$ in optimal Besov setting, under suitable smallness of the data. As regards the initial density, the admissible regularity is so low it may have (small) jumps across a $C^{1}$ interface. This is of particular interest from the viewpoint of physics as it implies that motion of a mixture of two incompressible fluids with slightly different densities can be modeled by the inhomogeneous Navier-Stokes equations. In addition, the regularity of the constructed velocity suffices to preserve the $C^{1}$ regularity of the interface between fluids.

We now come to the plan of the paper. In the next section, we present our main results and give some insight of the proof. Section 2 is devoted to solving the linearized system (0.1) in Lagrangian coordinates. This will enable us to define a map $\Phi: E_{p} \rightarrow E_{p}$ where $E_{p}$ stands for the functional space in which the Lagrangian version of the momentum equation of (0.1) is going to be solved. That $\Phi$ fulfills the conditions of the contraction mapping theorem on a small enough ball of $E_{p}$ is the main purpose of Section 3. In the Appendix we prove several important results concerning the Lagrangian coordinates and Besov spaces.

Notation: Throughout, the notation $C$ stands for a generic constant (the meaning of which depends on the context), and we sometimes write $X \lesssim Y$ instead of $X \leq C Y$. Finally, for $A=\left(A_{i j}\right)_{1 \leq i, j \leq n}$ and $B=\left(B_{i j}\right)_{1 \leq i, j \leq n}$ two matrices, we denote $A: B=\sum_{i, j} A_{i j} B_{j i}$. 


\section{MAin RESUlts AND PRINCIPLE OF THE PROOF}

Let us first derive formally the Lagrangian equations corresponding to $(0.1)^{1}$. Let $X_{u}$ be the flow associated to the vector-field $u$, that is the solution to

$$
X_{u}(t, y)=y+\int_{0}^{t} u\left(\tau, X_{u}(\tau, y)\right) d \tau .
$$

Let us recall that by Liouville's formula for transport equations, the divergence-free condition is equivalent to $\left|D X_{u}\right| \equiv 1$. In other words the map (1.1) is measure preserving. Now, denoting

$$
\bar{\rho}(t, y):=\rho\left(t, X_{u}(t, y)\right), \quad \bar{P}(t, y):=P\left(t, X_{u}(t, y)\right) \quad \text { and } \bar{u}(t, y)=u\left(t, X_{u}(t, y)\right)
$$

with $(\rho, u, \nabla P)$ a solution of (0.1), and using the chain rule and Lemma 1 from the Appendix, we gather that $\bar{\rho}(t, \cdot) \equiv \rho_{0}$ and that $(\bar{u}, \nabla \bar{P})$ satisfies

$$
\left\{\begin{array}{l}
\rho_{0} \partial_{t} \bar{u}-\mu \operatorname{div}_{y}\left(A_{u}{ }^{T} A_{u} \nabla_{y} \bar{u}\right)+{ }^{T} A_{u} \nabla_{y} \bar{P}=0 \quad \text { with } \quad A_{u}=\left(D_{y} X_{u}\right)^{-1}, \\
\operatorname{div}_{y}\left(A_{u} \bar{u}\right)=0 .
\end{array}\right.
$$

Motivated by prior works (see e.g. $[1,2,6,7]$ ) we want to solve the above system in critical homogeneous Besov spaces. Let us recall that, for $1 \leq p \leq \infty$ and $s \leq n / p$, a tempered distribution $u$ over $\mathbb{R}^{n}$ belongs to the homogeneous Besov space $\dot{B}_{p, 1}^{s}\left(\mathbb{R}^{n}\right)$ if

$$
u=\sum_{j \in \mathbb{Z}} \dot{\Delta}_{j} u \quad \text { in } \mathcal{S}^{\prime}\left(\mathbb{R}^{n}\right)
$$

and

$$
\|u\|_{\dot{B}_{p, 1}^{s}\left(\mathbb{R}^{n}\right)}:=\sum_{j \in \mathbb{Z}} 2^{j s}\left\|\dot{\Delta}_{j} u\right\|_{L_{p}\left(\mathbb{R}^{n}\right)}<\infty .
$$

Here $\left(\dot{\Delta}_{j}\right)_{j \in \mathbb{Z}}$ denotes a homogeneous dyadic resolution of unity in Fourier variables (see e.g. [4], Chap. 2 for more details).

Loosely speaking, a function belongs to $\dot{B}_{p, 1}^{s}\left(\mathbb{R}^{n}\right)$ if it as $s$ derivatives in $L_{p}\left(\mathbb{R}^{n}\right)$. In the present paper, we shall make an extensive use of the following classical properties:

- the Besov space $\dot{B}_{p, 1}^{n / p}\left(\mathbb{R}^{n}\right)$ is a Banach algebra embedded in the set of continuous functions going to 0 at infinity, whenever $1 \leq p<\infty$;

- the usual product maps $\dot{B}_{p, 1}^{n / p-1}\left(\mathbb{R}^{n}\right) \times \dot{B}_{p, 1}^{n / p}\left(\mathbb{R}^{n}\right)$ in $\dot{B}_{p, 1}^{n / p-1}\left(\mathbb{R}^{n}\right)$ whenever $1 \leq p<2 n$.

From now on, we shall omit $\mathbb{R}^{n}$ in the notation for Besov spaces. We shall obtain the existence and uniqueness of a global solution $(\bar{u}, \nabla \bar{P})$ for $(1.2)$ in the space

$$
E_{p}:=\left\{(\bar{u}, \nabla \bar{P}): \bar{u} \in \mathcal{C}_{b}\left(\mathbb{R}_{+} ; \dot{B}_{p, 1}^{n / p-1}\right), \partial_{t} \bar{u}, \nabla^{2} \bar{u}, \nabla \bar{P} \in L_{1}\left(\mathbb{R}_{+} ; \dot{B}_{p, 1}^{n / p-1}\right)\right\},
$$

and we shall endow $E_{p}$ with the norm

$$
\|(\bar{u}, \nabla \bar{P})\|_{E_{p}}:=\|\bar{u}\|_{L_{\infty}\left(\mathbb{R}_{+} ; \dot{B}_{p, 1}^{n / p-1}\right)}+\left\|\partial_{t} \bar{u}, \mu \nabla^{2} \bar{u}, \nabla \bar{P}\right\|_{L_{1}\left(\mathbb{R}_{+} ; \dot{B}_{p, 1}^{n / p-1}\right)} .
$$

We shall also use the local version $E_{p}(T)$ of $E_{p}$, pertaining to functions defined on $[0, T) \times \mathbb{R}^{n}$. Writing out the exact definition and the corresponding norm is left to the reader.

The required regularity for the initial density $\rho_{0}$ is that it belongs to the multiplier space $\mathcal{M}\left(\dot{B}_{p, 1}^{n / p-1}\right)$ for $\dot{B}_{p, 1}^{n / p-1}$, that is the set of those distributions $\rho_{0}$ such that $\psi \rho_{0}$ is in $\dot{B}_{p, 1}^{n / p-1}$ whenever $\psi$ is in $\dot{B}_{p, 1}^{n / p-1}$, endowed with the norm

$$
\left\|\rho_{0}\right\|_{\mathcal{M}\left(\dot{B}_{p, 1}^{n / p-1}\right)}:=\sup \left\|\psi \rho_{0}\right\|_{\dot{B}_{p, 1}^{n / p-1}}
$$

where the supremum is taken over those functions $\psi$ in $\dot{B}_{p, 1}^{n / p-1}$ with norm 1 .

\footnotetext{
${ }^{1}$ The reader may refer to the Appendix for the rigorous derivation in our functional setting.
} 
Let us now state our main result.

Theorem 1. Let $p \in[1,2 n)$ and $u_{0}$ be a divergence-free vector field in $\dot{B}_{p, 1}^{n / p-1}\left(\mathbb{R}^{n}\right)$. Assume that the initial density $\rho_{0}$ belongs to the multiplier space $\mathcal{M}\left(\dot{B}_{p, 1}^{n / p-1}\right)$. There exists a constant $c$ depending only on $p$ and on $n$ such that if

$$
\left\|\rho_{0}-1\right\|_{\mathcal{M}\left(\dot{B}_{p, 1}^{n / p-1}\right)}+\mu^{-1}\left\|u_{0}\right\|_{\dot{B}_{p, 1}^{n / p-1}} \leq c
$$

then System (1.2) has a unique global solution $(\bar{u}, \nabla \bar{P})$ in $E_{p}$. Moreover, we have

$$
\|(\bar{u}, \nabla \bar{P}) \leq C\| u_{0} \|_{\dot{B}_{p, 1}^{n / p-1}}
$$

for some constant $C$ depending only on $n$ and on $p$, and the flow map $\left(\rho_{0}, u_{0}\right) \longmapsto(\bar{u}, \nabla \bar{P})$ is Lipschitz continuous from $\mathcal{M}\left(\dot{B}_{p, 1}^{n / p-1}\right) \times \dot{B}_{p, 1}^{n / p-1}$ to $E_{p}$.

In the case where only the density satisfies the smallness condition, we get the following local-in-time existence result:

Theorem 2. Under the above regularity assumptions, there exists a constant $c$ depending only on $p$ and on $n$ such that if

$$
\left\|\rho_{0}-1\right\|_{\mathcal{M}\left(\dot{B}_{p, 1}^{n / p-1}\right)} \leq c
$$

then there exists some $T>0$ such that System (1.2) has a unique local solution $(\bar{u}, \nabla \bar{P})$ in $E_{p}(T)$, and the flow map $\left(\rho_{0}, u_{0}\right) \longmapsto(\bar{u}, \nabla \bar{P})$ is Lipschitz continuous from $\mathcal{M}\left(\dot{B}_{p, 1}^{n / p-1}\right) \times \dot{B}_{p, 1}^{n / p-1}$ to $E_{p}(T)$.

The regularity given by Theorem 1 ensures that the map defined in (1.1) is defined globally (see the Appendix). Coming back to the Eulerian formulation, this will enable us to get the following result ${ }^{2}$ :

Theorem 3. Under the above assumptions, System (0.1) has a unique global solution $(\rho, u, \nabla P)$ with $\rho \in L_{\infty}\left(\mathbb{R}_{+} ; \mathcal{M}\left(\dot{B}_{p, 1}^{n / p-1}\right)\right)$ and $(u, \nabla P) \in E_{p}$.

Let us make a few comments concerning the above assumptions.

- The condition $1 \leq p<2 n$ is a consequence of the product laws in Besov spaces. Let us emphasize that any space $L_{\infty} \cap B_{q, \infty}^{n / q-1}$ with $q$ satisfying

$$
\frac{1}{q}>\frac{1}{n}-\frac{1}{p} \text { and } \frac{1}{q} \geq \frac{1}{p}-\frac{1}{n}
$$

embeds in $\mathcal{M}\left(\dot{B}_{p, 1}^{n / p-1}\right)$, a consequence of basic continuity results for the paraproduct operator (see [4]). Hence the above statement improves those of $[1,2]$ as regards the uniqueness. In particular, one may take the initial velocity in a Besov space with a negative index of regularity, so that a highly oscillating "large" velocity may give rise to a unique global solution.

- In contrast with the results of $[1,2,6]$ it is not clear that the above statements may be generalized as so if the viscosity depends on the density. Recall that in this case, the diffusion term in the momentum equation of $(0.1)$ reads $\operatorname{div}\left(\mu(\rho)\left(\nabla u+{ }^{T} \nabla u\right)\right)$ where $\mu$ is a given suitably smooth nonnegative function. One may easily extend the above statements to this case under the stronger condition that $\left(\mu\left(\rho_{0}\right)-\mu(1)\right)$ is small in $\mathcal{M}\left(\dot{B}_{p, 1}^{n / p}\right)$.

\footnotetext{
${ }^{2}$ We here consider only the case of small data to simplify the presentation.
} 
The space $\mathcal{M}\left(\dot{B}_{p, 1}^{n / p-1}\right)$ is in fact much larger than $L_{\infty} \cap B_{q, \infty}^{n / q-1}$ with $q$ satisfying (1.4) (see e.g. [13], Chap. 4). It contains characteristic functions of $C^{1}$ bounded domains, whenever $p>n-1$ (see the proof in Lemma 5). Hence, our result applies to mixture of fluids, which is of course of great physical interest. In addition, given that the constructed velocity field $u$ is divergence-free and admits a $C^{1}$ flow $X$ (see again the appendix), we deduce the following result which emphasizes the range of Theorem 1 (we just state the case of small velocities to simplify the presentation):

Corollary 1. Assume that $u_{0} \in \dot{B}_{p, 1}^{n / p-1}$ with $\operatorname{div} u_{0}=0$ and $n-1<p<2 n$. Let $\Omega_{0}$ be a bounded $C^{1}$ domain of $\mathbb{R}^{n}$. There exist two constants $c$ (depending only on $p$ and $n$ ) and $c^{\prime}$ (depending only on $p, n$ and $\Omega_{0}$ ) such that if

$$
\left\|u_{0}\right\|_{\dot{B}_{p, 1}^{n / p-1}} \leq c \quad \text { and } \quad \rho_{0}=1+\sigma \chi_{\Omega_{0}} \text { with }|\sigma| \leq c^{\prime},
$$

then System (0.1) has a unique global solution $(\rho, u, \nabla P)$ with $\rho \in L_{\infty}\left(\mathbb{R}_{+} ; \mathcal{M}\left(\dot{B}_{p, 1}^{n / p-1}\right)\right)$ and $(u, \nabla P) \in E_{p}$. In addition, for all time,

$$
\rho(t)=1+\sigma \chi_{\Omega_{t}} \text { where } \Omega_{t}=X_{u}\left(t, \Omega_{0}\right) .
$$

Besides, the measure and the $C^{1}$ regularity of $\partial \Omega_{t}$ are preserved for all time.

Let us give the main ideas of the proof of existence. Obviously, it suffices to find a fixed point for the map $\Theta:(q, v) \mapsto(\rho, u)$ where $(\rho, u)$ stands for the solution to the linear system

$$
\left\{\begin{array}{l}
\partial_{t} \rho+v \cdot \nabla \rho=0, \\
q\left(\partial_{t} u+v \cdot \nabla u\right)-\mu \Delta u+\nabla P=0, \\
\operatorname{div} u=0, \\
\left.u\right|_{\mid t=0}=u_{0} .
\end{array}\right.
$$

Although it is possible to prove uniform estimates in $L_{\infty}\left(\mathbb{R}_{+} ; \mathcal{M}\left(\dot{B}_{p, 1}^{n / p-1}\right)\right) \times E_{p}$ for $(\rho, u, \nabla P)$, we do not know how to get stability estimates in the same space, owing to the hyperbolic nature of the density equation. As a consequence, the contraction mapping theorem does not apply.

In the present paper, we shall rather define the solution of the above system in the Lagrangian coordinates corresponding to $v$. For such coordinates, the density is time-independent. So, given some reference vector field $\bar{v}$ and pressure field $\nabla \bar{Q}$ with $(\bar{v}, \nabla \bar{Q}) \in E_{p}$, one may define $(\bar{u}, \nabla \bar{P})$ to be the solution in the Lagrangian coordinates $y=X_{v}^{-1}(t, x)$ pertaining to $v$ of the linear system (1.7) (that $X_{v}$ is a $C^{1}$-diffeomorphism over $\mathbb{R}^{n}$ is proved in the appendix).

Let us give more details. We assume that $\left|D X_{v}\right| \equiv 1$ and set

$$
\bar{\rho}(t, y):=\rho\left(t, X_{v}(t, y)\right), \quad \bar{P}(t, y):=P\left(t, X_{v}(t, y)\right) \text { and } \bar{u}(t, y)=u\left(t, X_{v}(t, y)\right),
$$

where $(\rho, u, \nabla P)$ stands for a solution to (1.7). Then we have $\bar{\rho}(t, \cdot) \equiv \rho_{0}$ and (see the proof in appendix)

with

$$
\begin{aligned}
& \rho_{0} \partial_{t} \bar{u}-\mu \operatorname{div}\left(A_{v}{ }^{T} A_{v} \nabla_{y} \bar{u}\right)+{ }^{T} A_{v} \nabla_{y} \bar{P}=0 \\
& \operatorname{div}_{y}\left(A_{v} \bar{u}\right)=0
\end{aligned}
$$

$$
A_{v}=\left(D_{y} X_{v}\right)^{-1} \quad \text { and } \quad X_{v}(t, y)=y+\int_{0}^{t} \bar{v}(\tau, y) d \tau
$$

Solving this linear system globally turns out to be possible under some smallness condition over $\bar{v}$ and $\rho_{0}-1$. This will enable us to define a self-map $\Phi:(\bar{v}, \nabla \bar{Q}) \mapsto(\bar{u}, \nabla \bar{P})$ on $E_{p}$. Then it will be only a matter of checking that if the data $\rho_{0}$ and $u_{0}$ satisfy a suitable smallness condition then the map $\Phi$ fulfills the assumptions of the standard Banach fixed point theorem. 
The key to that will be estimates for the Stokes system in $E_{p}$ (see Proposition 1) and a "magic" algebraic relation involving the second equation of (1.8) (which has been used before in e.g. $[14,15]$ in a different context).

\section{THE LINEAR THEORY}

Our proof of existence for the linear System (1.8) will be based on the following a priori estimates for the Stokes system, the proof of which may be found in $[7]^{3}$ :

Proposition 1. Let $p \in[1, \infty]$ and $s \in \mathbb{R}$. Let $u_{0} \in \dot{B}_{p, 1}^{s}\left(\mathbb{R}^{n}\right)$ and $f \in L_{1}\left(0, T ; \dot{B}_{p, 1}^{s}\left(\mathbb{R}^{n}\right)\right)$. Let $g:[0, T] \times \mathbb{R}^{n} \rightarrow \mathbb{R}$ be such that

$$
\nabla g \in L_{1}\left(0, T ; \dot{B}_{p, 1}^{s}\left(\mathbb{R}^{n}\right)\right), \quad \partial_{t} g=\operatorname{div} R \quad \text { with } R \in L_{1}\left(0, T ; \dot{B}_{p, 1}^{s}\left(\mathbb{R}^{n}\right)\right)
$$

and that the compatibility condition $\left.g\right|_{t=0}=\operatorname{div} u_{0}$ on $\mathbb{R}^{n}$ is satisfied.

Then System

$$
\begin{array}{lrr}
\partial_{t} u-\mu \Delta u+\nabla P=f & \text { in } & (0, T) \times \mathbb{R}^{n} \\
\operatorname{div} u=g & \text { in } & (0, T) \times \mathbb{R}^{n} \\
\left.u\right|_{t=t_{0}}=u_{0} & \text { on } & \mathbb{R}^{n}
\end{array}
$$

has a unique solution $(u, \nabla P)$ with

$$
u \in \mathcal{C}\left([0, T) ; \dot{B}_{p, 1}^{s}\left(\mathbb{R}^{n}\right)\right) \quad \text { and } \quad \partial_{t} u, \nabla^{2} u, \nabla P \in L_{1}\left(0, T ; \dot{B}_{p, 1}^{s}\left(\mathbb{R}^{n}\right)\right)
$$

and the following estimate is valid:

$$
\begin{aligned}
\|u\|_{L_{\infty}\left(0, T ; \dot{B}_{p, 1}^{s}\left(\mathbb{R}^{n}\right)\right)}+\| \partial_{t} u, \mu \nabla^{2} u, & \nabla P \|_{L_{1}\left(0, T ; \dot{B}_{p, 1}^{s}\left(\mathbb{R}^{n}\right)\right)} \\
& \leq C\left(\|f, \mu \nabla g, R\|_{L_{1}\left(0, T ; \dot{B}_{p, 1}^{s}\left(\mathbb{R}^{n}\right)\right)}+\left\|u_{0}\right\|_{\dot{B}_{p, 1}^{s}\left(\mathbb{R}^{n}\right)}\right)
\end{aligned}
$$

where $C$ is an absolute constant with no dependence on $\mu$ and $T$.

Granted with the above statement, we rewrite System (1.8) as

$$
\begin{aligned}
& \partial_{t} \bar{u}-\mu \Delta \bar{u}+\nabla \bar{P}=\left(1-\rho_{0}\right) \partial_{t} \bar{u}+\mu \operatorname{div}\left(\left(A_{v}{ }^{T} A_{v}-\mathrm{Id}\right) \nabla \bar{u}\right)+\left(\operatorname{Id}-{ }^{T} A_{v}\right) \nabla \bar{P} \\
& \operatorname{div} \bar{u}=\operatorname{div}\left(\left(\operatorname{Id}-A_{v}\right) \bar{u}\right) \\
& \left.\bar{u}\right|_{t=0}=u_{0} .
\end{aligned}
$$

We assume that the vector-field $\bar{v}$ from which $A_{v}$ and $D X_{v}$ are defined satisfies

$$
\bar{v} \in \mathcal{C}\left(\mathbb{R}_{+} ; \dot{B}_{p, 1}^{n / p-1}\right), \quad \partial_{t} \bar{v}, \nabla^{2} \bar{v} \in L_{1}\left(\mathbb{R}_{+} ; \dot{B}_{p, 1}^{n / p-1}\right), \quad\left|D X_{v}\right| \equiv 1
$$

and that, for a small enough constant $c$,

$$
\int_{0}^{\infty}\|D \bar{v}\|_{\dot{B}_{p, 1}^{n / p}} d t \leq c .
$$

Even though this system is linear, it cannot be solved directly by means of Proposition 1 for the right-hand side depends on the solution itself. So in order to prove the existence of $\bar{u}$, we shall look for a fixed point of the map

$$
\Psi:(\bar{w}, \nabla \bar{Q}) \longmapsto(\bar{u}, \nabla \bar{P})
$$

\footnotetext{
${ }^{3}$ Because homogeneous 0 -th order multipliers act on any homogeneous Besov spaces $\dot{B}_{p, 1}^{s}$, one may take any indices $s$ and exponents $p \in[1, \infty]$, in Proposition 1 .
} 
where $(\bar{w}, \nabla \bar{Q}) \in E_{p}$ and $(\bar{u}, \nabla \bar{P})$ stands for the solution of

$$
\left\{\begin{array}{l}
\partial_{t} \bar{u}-\mu \Delta \bar{u}+\nabla \bar{P}=f(\bar{w}, \nabla \bar{Q}), \\
\operatorname{div} \bar{u}=g(\bar{w}) \\
\left.\bar{u}\right|_{t=0}=u_{0} .
\end{array}\right.
$$

Above, $g(\bar{w}):=\operatorname{div}\left(\left(\operatorname{Id}-A_{v}\right) \bar{w}\right)$ and

$$
f(\bar{w}, \nabla \bar{Q}):=\left(1-\rho_{0}\right) \partial_{t} \bar{w}+\mu \operatorname{div}\left(\left(A_{v}{ }^{T} A-\operatorname{Id}\right) \nabla \bar{w}\right)+\left(\operatorname{Id}-{ }^{T} A_{v}\right) \nabla \bar{Q} .
$$

We claim that, if $\bar{v}$ satisfies (2.4) and the smallness condition (2.5), then for any $(\bar{w}, \nabla \bar{Q})$ in the space $E_{p}$ with $1 \leq p<2 n$, the above system has a unique solution $(\bar{u}, \nabla \bar{P})$ in $E_{p}$ and that, in addition, the map $\Psi$ fulfills the required conditions for applying the contracting mapping theorem.

The existence of $(\bar{u}, \nabla \bar{P})$ will stem from Proposition 1 once it has been checked that $f(\bar{w}, \nabla \bar{Q})$ and $g(\bar{w})$ fulfill the required conditions. As regards $g(\bar{w})$, this stems from the following "magic formula"

$$
g(\bar{w})=\operatorname{div}\left(\left(\operatorname{Id}-A_{v}\right) \bar{w}\right)=D \bar{w}:\left(\operatorname{Id}-A_{v}\right),
$$

a consequence of the fact that $\left|D X_{v}\right| \equiv 1$ (see Corollary 2 in the Appendix).

Bounds for $g(\bar{w})$. Let us first check that $g(\bar{w}) \in L_{1}\left(\mathbb{R}_{+} ; \dot{B}_{p, 1}^{n / p}\right)$. As $D \bar{w} \in L_{1}\left(\mathbb{R}_{+} ; \dot{B}_{p, 1}^{n / p}\right)$ and as, according to (A.8), Id $-A_{v}$ is in $L_{\infty}\left(\mathbb{R}_{+} ; \dot{B}_{p, 1}^{n / p}\right)$, this is a consequence of the fact that $\dot{B}_{p, 1}^{n / p}$ is a Banach algebra and that

$$
g(\bar{w})=D \bar{w}:\left(\operatorname{Id}-A_{v}\right)
$$

In addition, we get

$$
\|g(\bar{w})\|_{L_{1}\left(\mathbb{R}_{+} ; \dot{B}_{p, 1}^{n / p}\right)} \lesssim\|D \bar{v}\|_{L_{1}\left(\mathbb{R}_{+} ; \dot{B}_{p, 1}^{n / p}\right)}\|D \bar{w}\|_{L_{1}\left(\mathbb{R}_{+} ; \dot{B}_{p, 1}^{n / p}\right)} \cdot
$$

Next, we see that $\partial_{t}(g(\bar{w}))=\operatorname{div} R^{1}(\bar{w})+\operatorname{div} R^{2}(\bar{w})$ with

$$
R^{1}(\bar{w}):=\left(\operatorname{Id}-A_{v}\right) \partial_{t} \bar{w} \quad \text { and } \quad R^{2}(\bar{w}):=-\partial_{t} A_{v} \bar{w} .
$$

So, according to (A.7),(A.9) and because the product operator maps $\dot{B}_{p, 1}^{n / p} \times \dot{B}_{p, 1}^{n / p-1}$ in $\dot{B}_{p, 1}^{n / p-1}$ whenever $p<2 n$, we see that $R^{1}(\bar{w})$ and $R^{2}(\bar{w})$ belong to $L_{1}\left(\mathbb{R}_{+} ; \dot{B}_{p, 1}^{n / p-1}\right)$ and that

$$
\begin{aligned}
\left\|R^{1}(\bar{w})\right\|_{L_{1}\left(\mathbb{R}_{+} ; \dot{B}_{p, 1}^{n / p-1}\right)} & \lesssim\|D \bar{v}\|_{L_{1}\left(\mathbb{R}_{+} ; \dot{B}_{p, 1}^{n / p}\right)}\left\|\partial_{t} \bar{w}\right\|_{L_{1}\left(\mathbb{R}_{+} ; \dot{B}_{p, 1}^{n / p-1}\right)}, \\
\left\|R^{2}(\bar{w})\right\|_{L_{1}\left(\mathbb{R}_{+} ; \dot{B}_{p, 1}^{n / p-1}\right)} & \lesssim\|D \bar{v}\|_{L_{1}\left(\mathbb{R}_{+} ; \dot{B}_{p, 1}^{n / p}\right)}\|\bar{w}\|_{L_{\infty}\left(\mathbb{R}_{+} ; \dot{B}_{p, 1}^{n / p-1}\right)^{n}}
\end{aligned}
$$

Bounds for $f(\bar{w}, \nabla \bar{Q})$. That the first term of $f(\bar{w})$ belongs to $L_{1}\left(\mathbb{R}_{+} ; \dot{B}_{p, 1}^{n / p-1}\right)$ is a consequence of the definition of the multiplier space $\mathcal{M}\left(\dot{B}_{p, 1}^{n / p-1}\right)$; in addition we have

$$
\left\|\left(1-\rho_{0}\right) \partial_{t} \bar{w}\right\|_{\dot{B}_{p, 1}^{n / p-1}} \leq\left\|1-\rho_{0}\right\|_{\mathcal{M}\left(\dot{B}_{p, 1}^{n / p-1}\right)}\left\|\partial_{t} \bar{w}\right\|_{\dot{B}_{p, 1}^{n / p-1}} .
$$

Next, according to (A.11), the second term of $f(\bar{w})$ belongs to $L_{1}\left(\mathbb{R}_{+} ; \dot{B}_{p, 1}^{n / p-1}\right)$ and

$$
\left.\| \operatorname{div}\left(A_{v}^{T} A_{v}-\mathrm{Id}\right) \nabla \bar{w}\right)\left\|_{L_{1}\left(\mathbb{R}_{+} ; \dot{B}_{p, 1}^{n / p-1}\right)} \lesssim\right\| D \bar{v}\left\|_{L_{1}\left(\mathbb{R}_{+} ; \dot{B}_{p, 1}^{n / p}\right)}\right\| D \bar{w} \|_{L_{1}\left(\mathbb{R}_{+} ; \dot{B}_{p, 1}^{n / p}\right)} .
$$

Finally, Inequality (A.8) and the fact that the product operator maps $\dot{B}_{p, 1}^{n / p} \times \dot{B}_{p, 1}^{n / p-1}$ in $\dot{B}_{p, 1}^{n / p-1}$ if $p<2 n$ ensure that

$$
\left\|\left(\mathrm{Id}-{ }^{T} A_{v}\right) \nabla \bar{Q}\right\|_{L_{1}\left(\mathbb{R}_{+} ; \dot{B}_{p, 1}^{n / p-1}\right)} \lesssim\|\nabla \bar{v}\|_{L_{1}\left(\mathbb{R}_{+} ; \dot{B}_{p, 1}^{n / p}\right)}\|\nabla \bar{Q}\|_{L_{1}\left(\mathbb{R}_{+} ; \dot{B}_{p, 1}^{n / p-1}\right)} .
$$


So putting together (2.9) to (2.14), one may conclude from Proposition 1 that for any $(\bar{w}, \nabla \bar{Q})$ in $E_{p}$, System $(2.6)$ has a unique solution $(\bar{u}, \nabla \bar{P})$ in $E_{p}$. In addition

$$
\|(\bar{u}, \nabla \bar{P})\|_{E_{p}} \leq C\left(\left\|u_{0}\right\|_{\dot{B}_{p, 1}^{n / p-1}}+\left(\left\|1-\rho_{0}\right\|_{\mathcal{M}\left(\dot{B}_{p, 1}^{n / p-1}\right)}+\|D \bar{v}\|_{L_{1}\left(\mathbb{R}_{+} ; \dot{B}_{p, 1}^{n / p}\right)}\right)\|(\bar{w}, \nabla \bar{Q})\|_{E_{p}}\right) .
$$

As a consequence, there exists a positive constant $c$ (depending only on $n$ and on $p$ ) such that if $(2.5)$ is satisfied and

$$
\left\|1-\rho_{0}\right\|_{\mathcal{M}\left(\dot{B}_{p, 1}^{n / p-1}\right)} \leq c
$$

then

$$
\|\Psi(\bar{w}, \nabla \bar{Q})\|_{E_{p}} \leq C\left\|u_{0}\right\|_{\dot{B}_{p, 1}^{n / p-1}}+\frac{1}{2}\|(\bar{w}, \nabla \bar{Q})\|_{E_{p}} .
$$

Banach theorem thus entails that the linear map $\Psi$ admits a unique fixed point in $E_{p}$, that we shall still denote by $(\bar{u}, \nabla \bar{P})$. Let us emphasize that Inequality (2.16) ensures that

$$
\|(\bar{u}, \nabla \bar{P})\|_{E_{p}} \leq 2 C\left\|u_{0}\right\|_{\dot{B}_{p, 1}^{n / p-1}},
$$

and that, by construction, $\operatorname{div} A_{v} \bar{u}=0$ hence, according to Corollary $2,\left|D X_{u}\right| \equiv 1$. In other words, given $\bar{v}$ fulfilling (2.4) and (2.5), System (2.3) admits a unique solution $(\bar{u}, \nabla \bar{P})$ fulfilling the same conditions, together with (2.17).

\section{The inhomogeneous Navier-Stokes equations}

Let us denote by $\widetilde{E}_{p}^{R}$ the closed subset of $E_{p}$ containing all the couples $(\bar{v}, \nabla \bar{Q})$ such that

$$
\left|D X_{v}\right| \equiv 1 \quad \text { and } \quad\|(\bar{v}, \nabla \bar{Q})\|_{E_{p}} \leq R
$$

According to the previous section, if one takes $(\bar{v}, \nabla \bar{Q})$ in $E_{p}$ with $\bar{v}$ satisfying $\left|D X_{v}\right| \equiv 1$ and (2.5), then (1.8) admits a solution $(\bar{u}, \nabla \bar{P})$ in the same space, such that $\left|D X_{u}\right| \equiv 1$. Let $\Phi(\bar{v}, \nabla \bar{Q})$ denote this solution ${ }^{4}$. We claim that if $u_{0}$ is small enough with respect to $\mu$ in $\dot{B}_{p, 1}^{n / p-1}$, if the density $\rho_{0}$ satisfies (2.15) and if $R$ is small enough, then $\Phi$ admits a unique fixed point in $\widetilde{E}_{p}^{R}$, as a consequence of the contracting mapping theorem.

3.1. Stability of a small ball of $E_{p}$ by $\Phi$. Assume that (2.15) is satisfied and let us take $R=c \mu$. Then $(2.5)$ holds true whenever $(\bar{v}, \nabla \bar{Q})$ is in $\widetilde{E}_{p}^{R}$. Therefore $(\bar{u}, \nabla \bar{P}):=\Phi(\bar{v}, \nabla \bar{Q})$ satisfies (2.17). So it is clear that if

$$
2 C\left\|u_{0}\right\|_{\dot{B}_{p, 1}^{n / p-1}} \leq c \mu,
$$

with $C$ as in $(2.17)$, then $(\bar{u}, \nabla \bar{P})$ is in $\widetilde{E}_{p}^{R}$, too.

3.2. Contraction properties. In this part, we show that under Conditions (2.15) and (3.1) (with a greater constant $C$ and smaller constant $c$ if needed), the map $\Phi: \widetilde{E}_{p}^{R} \rightarrow \widetilde{E}_{p}^{R}$ is 1/2-Lipschitz.

So we are given $\left(\bar{v}_{1}, \nabla \bar{Q}_{1}\right)$ and $\left(\bar{v}_{2}, \nabla \bar{Q}_{2}\right)$ in $\widetilde{E}_{p}^{R}$, and denote

$$
\left(\bar{u}_{1}, \nabla \bar{P}_{1}\right):=\Phi\left(\bar{v}_{1}, \nabla \bar{Q}_{1}\right) \quad \text { and } \quad\left(\bar{u}_{2}, \nabla \bar{P}_{2}\right):=\Phi\left(\bar{v}_{2}, \nabla \bar{Q}_{2}\right) .
$$

Let $X_{1}$ and $X_{2}$ be the flows associated to $\bar{v}_{1}$ and $\bar{v}_{2}$. Set $A_{i}=\left(D X_{i}\right)^{-1}$ for $i=1,2$. The equations satisfied by $\delta u:=\bar{u}_{2}-\bar{u}_{1}$ and $\nabla \delta P:=\nabla \bar{P}_{2}-\nabla \bar{P}_{1}$ read

$$
\left\{\begin{array}{l}
\partial_{t} \delta u-\mu \Delta \delta u+\nabla \delta P=\delta f:=\delta f_{1}+\delta f_{2}+\delta f_{3}+\mu \operatorname{div} \delta f_{4}+\mu \operatorname{div} \delta f_{5} \\
\operatorname{div} \delta u=\delta g:=\operatorname{div}\left(\left(\operatorname{Id}-A_{2}\right) \delta u+\left(A_{1}-A_{2}\right) \bar{u}_{1}\right)
\end{array}\right.
$$

\footnotetext{
${ }^{4}$ Of course, it is independent of $\nabla \bar{Q}$. However, prescribing the pressure is needed so as to define a map from a subset of $E_{p}$ to itself.
} 
with

$$
\begin{aligned}
\delta f_{1}:= & \left(1-\rho_{0}\right) \partial_{t} \delta u, \quad \delta f_{2}:=\left(\operatorname{Id}-{ }^{T} A_{2}\right) \nabla \delta P, \quad \delta f_{3}:={ }^{T}\left(A_{1}-A_{2}\right) \nabla \bar{P}_{1}, \\
& \delta f_{4}:=\left(A_{2}{ }^{T} A_{2}-A_{1}{ }^{T} A_{1}\right) \nabla \bar{u}_{1}, \quad \delta f_{5}:=\left(A_{2}{ }^{T} A_{2}-\mathrm{Id}\right) \nabla \delta u .
\end{aligned}
$$

Once again, bounding $(\delta u, \nabla \delta P)$ will stem from Proposition 1 , which ensures that, if $\partial_{t} \delta g=$ $\operatorname{div} \delta R$ then

$$
\|(\delta u, \nabla \delta P)\|_{E_{p}} \lesssim\|\delta f\|_{L_{1}\left(\mathbb{R}_{+} ; \dot{B}_{p, 1}^{n / p-1}\right)}+\mu\|\delta g\|_{L_{1}\left(\mathbb{R}_{+} ; \dot{B}_{p, 1}^{n / p}\right)}+\|\delta R\|_{L_{1}\left(\mathbb{R}_{+} ; \dot{B}_{p, 1}^{n / p-1}\right)} .
$$

So we have to estimate $\delta f_{1}, \delta f_{2}, \delta f_{3}$ and $\delta f_{4}, \delta f_{5}$ in $L_{1}\left(\mathbb{R}_{+} ; \dot{B}_{p, 1}^{n / p-1}\right)$ and $L_{1}\left(\mathbb{R}_{+} ; \dot{B}_{p, 1}^{n / p}\right)$, respectively. First, from the definition of the multiplier space $\mathcal{M}\left(\dot{B}_{p, 1}^{n / p-1}\right)$, we readily have

$$
\left\|\delta f_{1}\right\|_{L_{1}\left(\mathbb{R}_{+} ; \dot{B}_{p, 1}^{n / p-1}\right)} \leq\left\|\rho_{0}-1\right\|_{\mathcal{M}\left(\dot{B}_{p, 1}^{n / p-1}\right)}\left\|\partial_{t} \delta u\right\|_{L_{1}\left(\mathbb{R}_{+} ; \dot{B}_{p, 1}^{n / p-1}\right)} .
$$

Next, using Inequalities (A.8),(A.11), and product laws in Besov space yields

$$
\begin{aligned}
& \left\|\delta f_{2}\right\|_{L_{1}\left(\mathbb{R}_{+} ; \dot{B}_{p, 1}^{n / p-1}\right)} \lesssim\left\|D \bar{v}_{2}\right\|_{L_{1}\left(\mathbb{R}_{+} ; \dot{B}_{p, 1}^{n / p}\right)}\|D \delta P\|_{L_{1}\left(\mathbb{R}_{+} ; \dot{B}_{p, 1}^{n / p-1}\right)}, \\
& \left\|\delta f_{5}\right\|_{L_{1}\left(\mathbb{R}_{+} ; \dot{B}_{p, 1}^{n / p}\right)} \lesssim\left\|D \bar{v}_{2}\right\|_{L_{1}\left(\mathbb{R}_{+} ; \dot{B}_{p, 1}^{n / p}\right)}\|D \delta u\|_{L_{1}\left(\mathbb{R}_{+} ; \dot{B}_{p, 1}^{n / p}\right)} .
\end{aligned}
$$

Inequality (A.13) ensures that

$$
\left\|\delta f_{3}\right\|_{L_{1}\left(\mathbb{R}_{+} ; \dot{B}_{p, 1}^{n / p}\right)} \lesssim\|D \delta v\|_{L_{1}\left(\mathbb{R}_{+} ; \dot{B}_{p, 1}^{n / p}\right)}\left\|D \bar{P}_{1}\right\|_{L_{1}\left(\mathbb{R}_{+} ; \dot{B}_{p, 1}^{n / p-1}\right)}
$$

whereas Inequalities (A.13), (A.14) yield

$$
\left\|\delta f_{4}\right\|_{L_{1}\left(\mathbb{R}_{+} ; \dot{B}_{p, 1}^{n / p}\right)} \lesssim\|D \delta v\|_{L_{1}\left(\mathbb{R}_{+} ; \dot{B}_{p, 1}^{n / p}\right)}\left\|D \bar{u}_{1}\right\|_{L_{1}\left(\mathbb{R}_{+} ; \dot{B}_{p, 1}^{n / p}\right)} .
$$

In order to bound $\delta g$ in $L_{1}\left(\mathbb{R}_{+} ; \dot{B}_{p, 1}^{n / p}\right)$, we shall use the fact that, by construction,

$$
\operatorname{div} \bar{u}_{i}=\operatorname{div}\left(\left(\operatorname{Id}-A_{i}\right) \bar{u}_{i}\right)=D \bar{u}_{i}:\left(\operatorname{Id}-A_{i}\right) .
$$

Hence

$$
\delta g=D \delta u:\left(\mathrm{Id}-A_{2}\right)-D \bar{u}_{1}:\left(A_{2}-A_{1}\right) .
$$

Now, easy computations based on (A.8) and (A.13) yield

$$
\begin{aligned}
& \left\|D \delta u:\left(\operatorname{Id}-A_{2}\right)\right\|_{L_{1}\left(\mathbb{R}_{+} ; \dot{B}_{p, 1}^{n / p}\right)} \lesssim\left\|D \bar{v}_{2}\right\|_{L_{1}\left(\mathbb{R}_{+} ; \dot{B}_{p, 1}^{n / p}\right)}\|D \delta u\|_{L_{1}\left(\mathbb{R}_{+} ; \dot{B}_{p, 1}^{n / p}\right)}, \\
& \left\|D \bar{u}_{1}:\left(A_{2}-A_{1}\right)\right\|_{L_{1}\left(\mathbb{R}_{+} ; \dot{B}_{p, 1}^{n / p}\right)} \lesssim\left\|D \bar{u}_{1}\right\|_{L_{1}\left(\mathbb{R}_{+} ; \dot{B}_{p, 1}^{n / p}\right)}\|D \delta\|_{L_{1}\left(\mathbb{R}_{+} ; \dot{B}_{p, 1}^{n / p}\right)}
\end{aligned}
$$

Finally, to bound $\partial_{t} \delta g$, we decompose it into $\operatorname{div}\left(\delta R_{1}+\delta R_{2}+\delta R_{3}+\delta R_{4}\right)$ with

$$
\begin{array}{ll}
\delta R_{1}=-\partial_{t} A_{2} \delta u, & \delta R_{2}=\left(\operatorname{Id}-A_{2}\right) \partial_{t} \delta u, \\
\delta R_{3}=\partial_{t}\left(A_{1}-A_{2}\right) \bar{u}_{1}, & \delta R_{4}=\left(A_{1}-A_{2}\right) \partial_{t} \bar{u}_{1} .
\end{array}
$$

Using (A.7), (A.9) and product laws in Besov spaces, we see that

$$
\begin{aligned}
&\left\|\delta R_{1}\right\|_{L_{1}\left(\mathbb{R}_{+} ; \dot{B}_{p, 1}^{n / p-1}\right)} \lesssim\left\|D \bar{v}_{2}\right\|_{L_{1}\left(\mathbb{R}_{+} ; \dot{B}_{p, 1}^{n / p}\right)}\|\delta u\|_{L_{\infty}\left(\mathbb{R}_{+} ; \dot{B}_{p, 1}^{n / p-1}\right)}, \\
&\left\|\delta R_{2}\right\|_{L_{1}\left(\mathbb{R}_{+} ; \dot{B}_{p, 1}^{n / p-1}\right)} \lesssim\left\|D \bar{v}_{2}\right\|_{L_{1}\left(\mathbb{R}_{+} ; \dot{B}_{p, 1}^{n / p}\right)}\left\|\partial_{t} \delta u\right\|_{L_{1}\left(\mathbb{R}_{+} ; \dot{B}_{p, 1}^{n / p-1}\right)} .
\end{aligned}
$$

In order to bound $\delta R_{3}$, it suffices to take advantage of (A.15). We get

$$
\left\|\delta R_{3}\right\|_{L_{1}\left(\mathbb{R}_{+} ; \dot{B}_{p, 1}^{n / p-1}\right)} \lesssim\|D \delta\|_{L_{1}\left(\mathbb{R}_{+} ; \dot{B}_{p, 1}^{n / p}\right)}\left\|\bar{u}_{1}\right\|_{L_{\infty}\left(\mathbb{R}_{+} ; \dot{B}_{p, 1}^{n / p-1}\right)} .
$$

Finally, using again (A.14), we see that

$$
\left\|\delta R_{4}\right\|_{L_{1}\left(\mathbb{R}_{+} ; \dot{B}_{p, 1}^{n / p-1}\right)} \lesssim\|D \delta v\|_{L_{1}\left(\mathbb{R}_{+} ; \dot{B}_{p, 1}^{n / p}\right)}\left\|\partial_{t} \bar{u}_{1}\right\|_{L_{1}\left(\mathbb{R}_{+} ; \dot{B}_{p, 1}^{n / p-1}\right)} .
$$


One can now plug Inequalities (3.3) to (3.13) in (3.2). We end up with

$$
\begin{aligned}
\|(\delta u, \nabla \delta P)\|_{E_{p}} \leq C\left(\left\|1-\rho_{0}\right\|_{\mathcal{M}\left(\dot{B}_{p, 1}^{n / p-1}\right)}+\left\|D \bar{v}_{2}\right\|_{L_{1}\left(\mathbb{R}_{+} ; \dot{B}_{p, 1}^{n / p}\right)}\right)\|(\delta u, \nabla \delta P)\|_{E_{p}} \\
+C \mu^{-1}\left\|\left(\bar{u}_{1}, \nabla \bar{P}_{1}\right)\right\|_{E_{p}}\|(\delta v, \nabla \delta Q)\|_{E_{p}} .
\end{aligned}
$$

So we see that if (2.5) and (2.15) are satisfied for $\bar{v}_{1}, \bar{v}_{2}$ and $\rho_{0}$ with a small enough constant $c$, then we have

$$
\left.\|(\delta u, \nabla \delta P)\|_{E_{p}} \leq 2 C R \mu^{-1}\|(\delta v, \nabla \delta Q)\|_{E_{p}}\right) .
$$

Hence, the map $\Phi: \widetilde{E}_{p}^{R} \mapsto \widetilde{E}_{p}^{R}$ is $1 / 2$-Lipschitz whenever $R$ and the data have been chosen so that (2.15), (3.1) are satisfied and $4 C R \leq \mu$. This completes the proof of existence of a unique solution to System $(1.2)$ in $\widetilde{E}_{p}^{R}$.

3.3. Stability estimates in $E_{p}$. In this part, we want to prove stability estimates in $E_{p}$ for the solutions to (1.2). This will ensure both uniqueness and that the flow map is Lipschitz.

So we consider two initial divergence-free velocity fields $u_{0,1}$ and $u_{0,2}$ in $\dot{B}_{p, 1}^{n / p-1}$, and densities $\left(\rho_{0,1}, \rho_{0,2}\right)$ in $\mathcal{M}\left(\dot{B}_{p, 1}^{n / p-1}\right)$ satisfying $(2.15)$ and (3.1). We want to compare two solutions $\left(\bar{u}_{1}, \nabla \bar{P}_{1}\right)$ and $\left(\bar{u}_{2}, \nabla \bar{P}_{2}\right)$ in $E_{p}$ of System $(1.2)$, corresponding to data $\left(\rho_{0,1}, u_{0,1}\right)$ and $\left(\rho_{0,2}, u_{0,2}\right)$.

The proof is similar to that of the contractivity of $\Phi$ : we have to bound

$$
\delta U(t):=\|\delta u\|_{L_{\infty}\left(0, t ; \dot{B}_{p, 1}^{n / p-1}\right)}+\left\|\partial_{t} \delta u, \mu \nabla^{2} \delta u, \nabla \delta \Pi\right\|_{L_{1}\left(0, t ; \dot{B}_{p, 1}^{n / p-1}\right)}
$$

(with $\delta u:=\bar{u}_{2}-\bar{u}_{1}$ and $\nabla \delta P:=\nabla \bar{P}_{2}-\nabla \bar{P}_{1}$ ) in terms of $\left\|\delta u_{0}\right\|_{\dot{B}_{p, 1}^{n / p-1}}$ and $\left\|\delta \rho_{0}\right\|_{\mathcal{M}\left(\dot{B}_{p, 1}^{n / p-1}\right)}$.

Now, the system for $(\delta u, \nabla \delta P)$ reads

$$
\left\{\begin{array}{l}
\partial_{t} \delta u-\mu \Delta \delta u+\nabla \delta P=\delta f_{0}+\delta f_{1}+\delta f_{2}+\delta f_{3}+\mu \operatorname{div}\left(\delta f_{4}+\delta f_{5}\right), \\
\operatorname{div} \delta u=\operatorname{div}\left(\left(\operatorname{Id}-A_{2}\right) \delta u+\left(A_{1}-A_{2}\right) \bar{u}_{1}\right)=D \delta u:\left(\operatorname{Id}-A_{2}\right)+D \bar{u}_{1}:\left(A_{1}-A_{2}\right)
\end{array}\right.
$$

with $\delta f_{0}:=\delta \rho_{0} \partial_{t} \bar{u}_{1}$ and where $\delta f_{i}$ for $i \in\{1, \cdots, 5\}$ has been defined in the previous subsection. Of course, now the matrices $A_{1}$ and $A_{2}$ correspond to the vector-fields $\bar{u}_{1}$ and $\bar{u}_{2}$.

Using Proposition 1, we gather that for all $t \in[0, T)$

$$
\begin{aligned}
& \begin{aligned}
\delta(t) \lesssim\left\|\delta u_{0}\right\|_{\dot{B}_{p, 1}^{n / p-1}}+\sum_{i=0}^{3} \| & \delta f_{i}\left\|_{L_{1}\left(0, t ; \dot{B}_{p, 1}^{n / p-1}\right)}+\mu \sum_{i=4}^{5}\right\| \delta f_{i}\left\|_{L_{1}\left(0, t ; \dot{B}_{p, 1}^{n / p}\right)}+\sum_{i=1}^{4}\right\| \delta R_{i} \|_{L_{1}\left(0, t ; \dot{B}_{p, 1}^{n / p-1}\right)} \\
& +\mu\left\|D \delta u:\left(\operatorname{Id}-A_{2}\right)\right\|_{L_{1}\left(0, t ; \dot{B}_{p, 1}^{n / p}\right)}+\mu\left\|D \bar{u}_{1}:\left(A_{2}-A_{1}\right)\right\|_{L_{1}\left(0, t ; \dot{B}_{p, 1}^{n / p}\right)}
\end{aligned} \\
& \begin{aligned}
(3.14) &
\end{aligned}
\end{aligned}
$$

From the definition of the multiplier space $\mathcal{M}\left(\dot{B}_{p, 1}^{n / p-1}\right)$, we readily have

$$
\left\|\delta f_{0}\right\|_{\dot{B}_{p, 1}^{n / p-1}} \leq\left\|\delta \rho_{0}\right\|_{\mathcal{M}\left(\dot{B}_{p, 1}^{n / p-1}\right)}\left\|\partial_{t} \bar{u}_{1}\right\|_{\dot{B}_{p, 1}^{n / p-1}} .
$$

The other terms may be bounded as in the previous subsection. So we eventually conclude that for all $t \in[0, T)$

$$
\delta U(t) \leq \frac{1}{2} \delta U(t)+C\left(\left\|\delta \rho_{0}\right\|_{\mathcal{M}\left(\dot{B}_{p, 1}^{n / p-1}\right)}+\left\|\delta u_{0}\right\|_{\dot{B}_{p, 1}^{n / p-1}}\right)
$$

whenever, for $i=1,2$,

$$
\sup _{t \in[0, T]}\left\|\bar{u}_{i}(t)\right\|_{\dot{B}_{p, 1}^{n / p-1}}+\int_{0}^{T}\left(\mu^{-1}\left\|\partial_{t} \bar{u}_{i}, \nabla \bar{P}_{i}\right\|_{\dot{B}_{p, 1}^{n / p-1}}+\left\|D \bar{u}_{i}\right\|_{\dot{B}_{p, 1}^{n / p}}\right) d t
$$

is small enough.

This latter condition is a consequence of (3.1). This completes the proof of stability estimates. 
3.4. Proof of the local-in-time existence result. We here explain how the arguments of the previous subsections have to be modified so as to handle large initial velocities.

Let us first notice that the computations that have been performed in Section 2 also hold locally on $[0, T)$ whenever $\bar{v}$ satisfies

$$
\bar{v} \in \mathcal{C}_{b}\left([0, T) ; \dot{B}_{p, 1}^{n / p-1}\right), \quad \partial_{t} \bar{v}, \nabla^{2} \bar{v} \in L_{1}\left(0, T ; \dot{B}_{p, 1}^{n / p-1}\right), \quad\left|D X_{v}\right| \equiv 1 \quad \text { on }[0, T) \times \mathbb{R}^{n}
$$

and

$$
\int_{0}^{T}\|D \bar{v}\|_{\dot{B}_{p, 1}^{n / p}} d t \leq c
$$

This ensures that, under Condition (2.15), System (1.8) may be solved locally in $E_{p}(T)$. Of course, Inequality (2.17) is still satisfied (for the norm in $E_{p}(T)$ ). However, it is not accurate enough so as to solve the nonlinear system, if $u_{0}$ is too large. To overcome this, we shall apply the contracting mapping theorem in some suitable neighborhood of the solution $\left(u_{L}, \nabla P_{L}\right)$ to the "free" Stokes system, that is

$$
\begin{array}{lrr}
\partial_{t} u_{L}-\mu \Delta u_{L}+\nabla P_{L}=0 & \text { in } & {[0, T) \times \mathbb{R}^{n},} \\
\operatorname{div} u_{L}=0 & \text { in } & {[0, T) \times \mathbb{R}^{n},} \\
\left.u_{L}\right|_{t=0}=u_{0} & \text { on } & \mathbb{R}^{n} .
\end{array}
$$

Setting $(\bar{u}, \nabla \bar{P}):=\Phi(\bar{v}, \nabla \bar{Q})$, we want to show that if $T$ is small enough (a condition which will be expressed in terms of the free solution only) then $(\widetilde{u}, \nabla \widetilde{P}):=\left(\bar{u}-u_{L}, \nabla\left(\bar{P}-P_{L}\right)\right)$ is small. For that, we shall apply Proposition 1 to the system satisfied by $(\widetilde{u}, \nabla \widetilde{P})$, namely

$$
\begin{aligned}
& \partial_{t} \widetilde{u}-\mu \Delta \widetilde{u}+\nabla \widetilde{P}=f(\bar{u}, \nabla \bar{P}) \\
& \operatorname{div} \widetilde{u}=g(\bar{u}), \\
& \left.\widetilde{u}\right|_{t=0}=0
\end{aligned}
$$

where $f(\bar{u}, \nabla \bar{P})$ and $g(\bar{u})$ have been defined in Section 2 .

On the one hand, we shall bound $f(\bar{u}, \nabla \bar{P})$ in $L_{1}\left(0, T ; \dot{B}_{p, 1}^{n / p-1}\right)$ and $g(\bar{u})$ in $L_{1}\left(0, T ; \dot{B}_{p, 1}^{n / p}\right)$ exactly as in Section 2, on the other hand, decomposing $\partial_{t}(g(\bar{u}))$ into $R^{1}(\bar{u})+R^{2}(\bar{u})$, we see that the bound (2.11) for $R^{2}(\bar{u})$ is not accurate enough as it involves $\|\bar{u}\|_{L_{\infty}\left(0, T ; \dot{B}_{p, 1}^{n / p-1}\right)}$ which need not be small for $T$ going to 0 , if $u_{0}$ is large. So we shall rather write

$$
R^{2}(\bar{u})=-\partial_{t} A_{v} u_{L}-\partial_{t} A_{v} \widetilde{u},
$$

and use product laws and Inequality (A.10), to get

$$
\left\|R^{2}(\bar{u})\right\|_{L_{1}\left(0, T ; \dot{B}_{p, 1}^{n / p-1}\right)} \lesssim\|D \bar{v}\|_{L_{2}\left(0, T ; \dot{B}_{p, 1}^{n / p-1}\right)}\left\|u_{L}\right\|_{L_{2}\left(0, T ; \dot{B}_{p, 1}^{n / p}\right)}+\|D \bar{v}\|_{L_{1}\left(0, T ; \dot{B}_{p, 1}^{n / p}\right)}\|\widetilde{u}\|_{L_{\infty}\left(0, T ; \dot{B}_{p, 1}^{n / p-1}\right)} .
$$

So, finally, using Proposition 1 and decomposing everywhere $\bar{u}$ and $\nabla \bar{P}$ in $u_{L}+\widetilde{u}$ and $\nabla P_{L}+\nabla \widetilde{P}$, we get

$$
\begin{aligned}
& \|(\widetilde{u}, D \widetilde{P})\|_{E_{p}(T)} \leq C\left(\left\|1-\rho_{0}\right\|_{\mathcal{M}\left(\dot{B}_{p, 1}^{n / p-1}\right)}+\|D \bar{v}\|_{L_{1}\left(0, T ; \dot{B}_{p, 1}^{n / p}\right)}\right) \\
& \quad \times\left(\|(\widetilde{u}, D \widetilde{P})\|_{E_{p}(T)}+\left\|\partial_{t} u_{L}, \mu D^{2} u_{L}, D P_{L}\right\|_{L_{1}\left(0, T ; \dot{B}_{p, 1}^{n / p-1}\right)}\right)+C\|\bar{v}\|_{L_{2}\left(0, T ; \dot{B}_{p, 1}^{n / p}\right)}\left\|u_{L}\right\|_{L_{2}\left(0, T ; \dot{B}_{p, 1}^{n / p}\right)} .
\end{aligned}
$$

Therefore, if (2.15) and (3.16) are satisfied with $c$ small enough and denote $\widetilde{v}:=\bar{v}-u_{L}$, then we get

$$
\begin{aligned}
\|(\widetilde{u}, D \widetilde{P})\|_{E_{p}(T)} \leq C c \| \partial_{t} u_{L}, \mu D^{2} u_{L}, D P_{L} & \|_{L_{1}\left(0, T ; \dot{B}_{p, 1}^{n / p-1}\right)} \\
& +C\left\|u_{L}\right\|_{L_{2}\left(0, T ; \dot{B}_{p, 1}^{n / p}\right)}^{2}+C\|\widetilde{v}\|_{L_{2}\left(0, T ; \dot{B}_{p, 1}^{n / p}\right)}\left\|u_{L}\right\|_{L_{2}\left(0, T ; \dot{B}_{p, 1}^{n / p}\right)} .
\end{aligned}
$$


This inequality together with the interpolation inequality

$$
\|\widetilde{v}\|_{L_{2}\left(0, T ; \dot{B}_{p, 1}^{n / p}\right)}\|\leq\| \widetilde{v}\left\|_{L_{1}\left(0, T ; \dot{B}_{p, 1}^{n / p+1}\right)}^{1 / 2}\right\| \widetilde{v} \|_{L_{\infty}\left(0, T ; \dot{B}_{p, 1}^{n / p-1}\right)}^{1 / 2}
$$

ensures that $\Phi$ maps $\left(u_{L}, \nabla P_{L}\right)+\widetilde{E}_{p}^{R}(T)$ (where $\widetilde{E}_{p}^{R}(T)$ is the "local" version of $\widetilde{E}_{p}^{R}$ ) into itself whenever $T$ satisfies

$$
\begin{aligned}
& C c\left\|\partial_{t} u_{L}, \mu D^{2} u_{L}, D P_{L}\right\|_{L_{1}\left(0, T ; \dot{B}_{p, 1}^{n / p-1}\right)}+C\left\|u_{L}\right\|_{L_{2}\left(0, T ; \dot{B}_{p, 1}^{n / p}\right)}^{2} \leq R / 2, \\
& C \mu^{-1 / 2}\left\|u_{L}\right\|_{L_{2}\left(0, T ; \dot{B}_{p, 1}^{n / p}\right)} \leq 1 / 2 .
\end{aligned}
$$

Of course, for (3.16) to be satisfied, it suffices that to take $R=c \mu / 2$ and to assume that $T$ is so small as

$$
\left\|D u_{L}\right\|_{L_{1}\left(0, T ; \dot{B}_{p, 1}^{n / p}\right)} \leq c / 2 .
$$

So if (3.19) and (3.20) are satisfied (conditions which depend only on the data) then one may conclude that $\Phi$ maps $\left(u_{L}, \nabla P_{L}\right)+\widetilde{E}_{p}^{R}(T)$ into itself.

The proof of the contraction properties for $\Phi$ in this context follows the same lines : we consider $\left(\bar{u}_{i}, \nabla \bar{P}_{i}\right)=\Phi\left(\bar{v}_{i}, \nabla \bar{Q}_{i}\right)$ with $\left(\bar{v}_{i}, \nabla \bar{Q}_{i}\right)$ in $\left(u_{L}, \nabla P_{L}\right)+\widetilde{E}_{p}^{R}(T)$ for $i=1,2$, then we bound all the terms $\delta f_{i}, \delta g_{i}$ and $\delta R_{i}$ as in the case of small initial velocity, except for $\delta R_{3}$ as it involves $\left\|\bar{u}_{1}\right\|_{L_{\infty}\left(0, T ; \dot{B}_{p, 1}^{n / p-1}\right)}$ which need not be small for $T$ going to 0 . For this latter term, we notice that, according to (A.16),

$$
\left\|\delta R_{3}\right\|_{L_{1}\left(0, T ; \dot{B}_{p, 1}^{n / p-1}\right)} \lesssim\|\delta v\|_{L_{2}\left(0, T ; \dot{B}_{p, 1}^{n / p}\right)}\left\|\bar{u}_{1}\right\|_{L_{2}\left(0, T ; \dot{B}_{p, 1}^{n / p}\right)} .
$$

So we eventually get,

$$
\begin{aligned}
\|(\delta u, \nabla \delta P)\|_{E_{p}(T)} \leq C(\| 1 & \left.-\rho_{0}\left\|_{\mathcal{M}\left(\dot{B}_{p, 1}^{n / p-1}\right)}+\right\| D \bar{v}_{2} \|_{L_{1}\left(0, T ; \dot{B}_{p, 1}^{n / p}\right)}\right)\|(\delta u, \nabla \delta P)\|_{E_{p}(T)} \\
& \left.+C\left(\mu^{-1}\left\|\left(\widetilde{u}_{1}, \nabla \widetilde{P}_{1}\right)\right\|_{E_{p}(T)}+\mu^{-1 / 2}\left\|u_{L}\right\|_{L_{2}\left(0, T ; \dot{B}_{p, 1}^{n / p}\right)}\right)\|(\delta v, \nabla \delta Q)\|_{E_{p}(T)}\right) .
\end{aligned}
$$

Note that our assumptions on $\left(\widetilde{u}_{1}, \nabla \widetilde{P}_{1}\right)$ and on the free solution ensures that if $R$ and $T$ have been chosen small enough then the factor of the last term is smaller than, say, $1 / 2$. So the contraction mapping theorem applies. This completes the proof of the existence part of Theorem 2. Proving the stability and uniqueness follows from similar arguments. The details are left to the reader.

3.5. Proof of Theorem 3. Given data $\left(\rho_{0}, u_{0}\right)$ satisfying the assumptions of Theorem 3, one may construct a global solution $(\bar{u}, \nabla \bar{P})$ to System (1.2) in $E_{p}$. If $X_{u}$ denotes the "flow" to $\bar{u}$ which is defined according to (1.9) then the results of the appendix ensure that, for all $t \in \mathbb{R}_{+}$, $X_{u}(t, \cdot)$ is a $C^{1}$ diffeomorphism of $\mathbb{R}^{n}$. In particular, one may set

$$
\rho(t, \cdot):=\rho_{0} \circ X_{u}^{-1}(t, \cdot), \quad P(t, \cdot):=\bar{P}(t, \cdot) \circ X_{u}^{-1}(t, \cdot), \quad u(t, \cdot):=\bar{u}(t, \cdot) \circ X_{u}^{-1}(t, \cdot),
$$

and the algebraic relations that are derived in the appendix show that $(\rho, u, \nabla P)$ satisfies System (0.1). In addition, given that $X_{u}(t, \cdot)$ is measure preserving and that $D X_{u}(t)-$ Id belongs to $\dot{B}_{p, 1}^{n / p}$, the map $a \mapsto a \circ X_{u}^{ \pm 1}(t)$ is continuous from $\dot{B}_{p, 1}^{s}$ to itself if $s \in\{n / p-1, n / p\}$, (see e.g. [8], Chap. 2). This implies that:

- the Eulerian velocity $u$ is in $\mathcal{C}_{b}\left(\mathbb{R}_{+} ; \dot{B}_{p, 1}^{n / p-1}\right)$,

- for any $\phi \in \dot{B}_{p, 1}^{n / p-1}$, we have $\phi \circ X_{u}^{ \pm 1}(t) \in \dot{B}_{p, 1}^{n / p-1}$. So, given that $\rho_{0} \in \mathcal{M}\left(\dot{B}_{p, 1}^{n / p-1}\right)$, we have

$$
\phi \rho(t)=\left(\left(\phi \circ X_{u}(t)\right) \rho_{0}\right) \circ X_{u}^{-1}(t) \in \dot{B}_{p, 1}^{n / p-1} .
$$

Hence $\rho \in L_{\infty}\left(\mathbb{R}_{+} ; \mathcal{M}\left(\dot{B}_{p, 1}^{n / p-1}\right)\right)$. 
- the chain rule ensures that

$$
\nabla P=\left({ }^{T} A_{u} \cdot \nabla \bar{P}\right) \circ X_{u}^{-1} .
$$

So combining product laws and the invariance of $\dot{B}_{p, 1}^{n / p-1}$ by right-composition, we get $\nabla P \in L_{1}\left(\mathbb{R}_{+} ; \dot{B}_{p, 1}^{n / p-1}\right)$.

- the chain rule also ensures that

$$
\nabla u=\left({ }^{T} A_{u} \cdot \nabla \bar{u}\right) \circ X_{u}^{-1} .
$$

So using the fact that $\dot{B}_{p, 1}^{n / p}$ is a Banach algebra, and using invariance of $\dot{B}_{p, 1}^{n / p}$ by rightcomposition, we get $\nabla u \in L_{1}\left(\mathbb{R}_{+} ; \dot{B}_{p, 1}^{n / p}\right)$.

In order to prove uniqueness, we consider two solutions $\left(\rho_{1}, u_{1}, \nabla P_{1}\right)$ and $\left(\rho_{2}, u_{2}, \nabla P_{2}\right)$ corresponding to the same data $\left(\rho_{0}, u_{0}\right)$, and perform the Lagrangian change of variable (pertaining to the flow of $u_{1}$ and $u_{2}$ respectively. The obtained functions $\left(\bar{u}_{1}, \nabla \bar{P}_{1}\right)$ and $\left(\bar{u}_{2}, \nabla \bar{P}_{2}\right)$ both satisfy (1.2) with the same $\rho_{0}$ and $u_{0}$. Hence they coincide, as a consequence of the uniqueness part of Theorem 1 .

\section{Appendix A. Appendix}

Let us first derive algebraic relations involving changes of coordinates.

We are given a $C^{1}$-diffeomorphism $X$ over $\mathbb{R}^{n}$. For $H: \mathbb{R}^{n} \rightarrow \mathbb{R}^{m}$, we agree that $\bar{H}(y)=$ $H(x)$ with $x=X(y)$. With this convention, the chain rule writes

$$
D_{y} \bar{H}(y)=D_{x} H(X(y)) \cdot D_{y} X(y) \quad \text { with }\left(D_{y} X\right)_{i j}=\partial_{y_{j}} X^{i} .
$$

or, denoting $\nabla_{y}={ }^{T} D_{y}$,

$$
\nabla_{y} \bar{H}(y)=\left(\nabla_{y} X(y)\right) \cdot \nabla_{x} H(X(y))
$$

Hence we have

$$
D_{x} H(x)=D_{y} \bar{H}(y) \cdot A(y) \quad \text { with } \quad A(y)=\left(D_{y} X(y)\right)^{-1}=D_{x} X^{-1}(x) .
$$

Lemma 1. Let $H$ be a vector-field over $\mathbb{R}^{n}$. If we denote $\bar{H}=H \circ X$ then the following relation holds true:

$$
\operatorname{div}_{x} H(x)=\operatorname{div}_{y}(A|D X| \bar{H})(y)=\operatorname{div}_{y}(\operatorname{adj}(D X) \bar{H})(y) \quad \text { with } \quad x=X(y)
$$

where $|D X|$ stands for the determinant of $D X$, and $\operatorname{adj}(D X)$ for the adjugate of $D X$, that is the transpose of the cofactor matrix of $D X$.

Proof: This stems from the following series of computations (based on integrations by parts and (A.2)) which hold for any scalar test function $q$ :

$$
\begin{aligned}
\int q(x) \operatorname{div}_{x} H(x) d x & =-\int D_{x} q(x) \cdot H(x) d x, \\
& =-\int D_{x} q(X(y)) \cdot H(X(y))\left|D_{y} X(y)\right| d y \\
& =-\int D_{y} \bar{q}(y) \cdot\left(A\left|D_{y} X\right|\right)(y) \cdot \bar{H}(y) d y, \\
& =\int \bar{q}(y) \operatorname{div}_{y}\left(A\left|D_{y} X\right| H\right)(y) d y .
\end{aligned}
$$

As $A=\left|D_{y} X\right|^{-1} \operatorname{adj}\left(D_{y} X\right)$, we get the result.

Remark 1. Combining (A.2) and (A.3), we deduce that if $a: \mathbb{R}^{n} \rightarrow \mathbb{R}$ then

$$
\overline{\Delta_{x} a}=\overline{\operatorname{div}_{x} \nabla_{x} a}=\operatorname{div}_{y}\left(A|D X| \overline{\nabla_{x} a}\right)=\operatorname{div}_{y}\left(\operatorname{adj}(D X)^{T} A \nabla_{y} \bar{a}\right) .
$$


Recall that if $v$ is a time-dependent vector field with coefficients in $L_{1}\left(0, T ; C^{0,1}\right)$ then it has, by virtue of the Cauchy-Lipschitz theorem, a unique $C^{1}$ flow $X_{v}$ satisfying

$$
X_{v}(t, y)=y+\int_{0}^{t} v\left(\tau, X_{v}(\tau, y)\right) d \tau \text { for all } t \in[0, T)
$$

and that $X_{v}(t, \cdot)$ is a $C^{1}$-diffeomorphism over $\mathbb{R}^{n}$.

Lemma 1 enables us to deduce the following "magic" relation which is the corner stone of the proof of our main results:

Corollary 2. Let $v$ and $w$ be two time-dependent vector fields with coefficients in $L_{1}\left(0, T ; C^{0,1}\right)$. Let $X_{v}$ and $X_{w}$ be the corresponding flows. Denote $A_{v}:=\left(D X_{v}\right)^{-1}$ and $A_{w}:=\left(D X_{w}\right)^{-1}$. Let us introduce the Lagrangian coordinates $y_{v}$ and $y_{w}$ pertaining to $v$ and $w$, respectively, defined by

$$
x=X_{v}\left(y_{v}\right)=X_{w}\left(y_{w}\right) .
$$

Assume in addition that

$$
\left|D X_{v}\right| \equiv 1 \text { and } \operatorname{div}\left(A_{v} \bar{w}_{v}\right)=0 \quad \text { with } \quad \bar{w}_{v}:=w \circ X_{v} .
$$

Then $\left|D X_{w}\right| \equiv 1$ and for any $C^{1}$ vector-field $H$, one has

$\operatorname{div} H(x)=\left(D \bar{H}_{v}: A_{v}\right)\left(y_{v}\right)=\operatorname{div}\left(A_{w} \bar{H}_{w}\right)\left(y_{w}\right)$ with $\bar{H}_{v}:=H \circ X_{v}$ and $\bar{H}_{w}:=H \circ X_{w}$.

Proof: With the above notation, the chain rule ensures that

$$
D_{x} H(x)=D_{y_{v}} \bar{H}_{v}\left(y_{v}\right) \cdot A_{v}\left(y_{v}\right) .
$$

Hence taking the trace yields the left equality.

Next, according to Lemma 1 and to our assumption over $v$ and $w$, we have

$$
0=\operatorname{div}\left(A_{v} \bar{w}_{v}\right)=\operatorname{div}\left(\operatorname{adj}\left(D X_{v}\right) \bar{w}_{v}\right)=\operatorname{div}_{x} w(x) .
$$

Hence Liouville theorem ensures that $\left|D X_{w}\right| \equiv 1$. So finally, applying Lemma 1 with $X_{w}$ completes the proof.

Lemma 2. There exist $n^{2}$ at least quadratic polynomials $P_{i j}: \mathcal{M}_{n}(\mathbb{R}) \rightarrow \mathbb{R}$ of degree $n-1$ such that

$$
\operatorname{Id}-\operatorname{adj}(\operatorname{Id}+C)=(C-(\operatorname{Tr} C) \operatorname{Id})+P_{2}(C),
$$

where $P_{2}(C)$ is the $n \times n$ matrix with entries $P_{i j}(C)$.

Proof: It suffices to use the fact that, by definition of the differential of adj, we have

$$
\operatorname{Id}-\operatorname{adj}(\operatorname{Id}+C)=\operatorname{adj}(\operatorname{Id})-\operatorname{adj}(\operatorname{Id}+C)=-d \operatorname{adj}(\operatorname{Id})(C)+P_{2}(C) .
$$

Now,

$$
\operatorname{adj}(\operatorname{Id}+C)=(\operatorname{Id}+C)^{-1} \operatorname{det}(\operatorname{Id}+C)
$$

and the differential of the reciprocal operator at Id is $C \mapsto-C$ while $d \operatorname{det}(\operatorname{Id})(C)=(\operatorname{Tr} C) \operatorname{Id}$. So $d \operatorname{adj}(\operatorname{Id})(C)=(\operatorname{Tr} C) \operatorname{Id}-C$.

We now want to establish some a priori estimates for the flow which will be needed in our main results. The first difficulty that has to be faced is that when implementing the iterative process for solving (1.2), we are given the velocity field $\bar{v}$ in Lagrangian coordinates. Therefore, it first has to be checked whether the "flow" $X_{v}(t, \cdot)$ defined by

$$
X_{v}(t, y):=y+\int_{0}^{t} \bar{v}(\tau, y) d \tau
$$

is a $C^{1}$ diffeomorphism over $\mathbb{R}^{n}$. This property is required for constructing the Eulerian vectorfield $v$ by setting $v(t, \cdot):=v \circ X_{v}^{-1}(t, \cdot)$. 
So let us assume that we are given some vector field $\bar{v}$ over $[0, T) \times \mathbb{R}^{n}$ with

$$
\bar{v} \in \mathcal{C}_{b}\left([0, T) ; \dot{B}_{p, 1}^{n / p-1}\right), \quad \partial_{t} \bar{v} \in L_{1}\left([0, T) ; \dot{B}_{p, 1}^{n / p-1}\right) \quad \text { and } \quad D \bar{v} \in L_{1}\left([0, T) ; \dot{B}_{p, 1}^{n / p}\right) .
$$

Differentiating (A.4) with respect to the space variable yields

$$
D X_{v}(t, y):=\operatorname{Id}+\int_{0}^{t} D \bar{v}(\tau, y) d \tau
$$

As $\dot{B}_{p, 1}^{n / p}\left(\mathbb{R}^{n}\right)$ is embedded in the set $\mathcal{C}_{0}\left(\mathbb{R}^{n}\right)$ of continuous functions going to 0 at infinity, we deduce that $X_{v}$ is a $C^{1}$ function over $\mathbb{R}_{+} \times \mathbb{R}^{n}$. However, in general, $X_{v}(t, \cdot)$ need not be a $C^{1}$-diffeomorphism over $\mathbb{R}^{n}$ for all $t \in \mathbb{R}_{+}$. So we assume that the smallness condition (2.5) is satisfied with $c$ small enough. Then, using embedding we see that it guarantees that

$$
\left\|D X_{v}(t, \cdot)-\operatorname{Id}\right\|_{L_{\infty}\left(\mathbb{R}^{n}\right)} \leq 1 / 2 \text { for all } t \in \mathbb{R}_{+} .
$$

Hence, for any $t \in \mathbb{R}_{+}$, the map $X_{v}(t, \cdot)$ is a local diffeomorphism. In order to show that it is a global diffeomorphism, we introduce the solution $Y_{v}$ to the ordinary differential equation

$$
\bar{v}\left(t, Y_{v}(t, x)\right)+D X_{v}\left(t, Y_{v}(t, x)\right) \frac{d}{d t} Y_{v}(t, x)=0 .
$$

Under (2.5), the matrix $D X_{v}$ is invertible at every point and $\left(D X_{v}\right)^{-1}-$ Id belongs to $L_{\infty}\left(\mathbb{R}_{+} ; \dot{B}_{p, 1}^{n / p}\right)$. Indeed, one may write

$$
\left(D X_{v}\right)^{-1}-\mathrm{Id}=\sum_{k \geq 1}\left(\mathrm{Id}-D X_{v}\right)^{k}
$$

Hence, using the assumptions over $\bar{v}$ and the product laws in Besov spaces (here we need that $1 \leq p<2 n$ ), (A.6) may be seen as an ordinary differential equation in the Banach space $\dot{B}_{p, 1}^{n / p-1}$, which may be solved on $[0, T)$ according to Cauchy-Lipschitz theorem. In particular, differentiating $X_{v}(t, \cdot) \circ Y_{v}(t, \cdot)$ with respect to time, we easily gather that $X_{v}(t, \cdot) \circ Y_{v}(t, \cdot)=\operatorname{Id}$ for all $t \in[0, T)$. Therefore, one may eventually conclude that $X_{v}(t, \cdot)$ is a $C^{1}$-diffeomorphism over $\mathbb{R}^{n}$, with inverse $Y_{v}(t, \cdot)$.

Let us now derive some "flow estimates" that will be needed for constructing the maps $\Phi$ and $\Psi$. For completeness, the statements that we here state are slightly more general than needed : that $|D X| \equiv 1$ is not assumed.

Lemma 3. Let $p \in[1,+\infty)$. Under Assumption (2.5) for $\bar{v}$, we have

$$
\begin{aligned}
& \|\operatorname{Id}-\operatorname{adj}(D X(t))\|_{\dot{B}_{p, 1}^{n / p}} \lesssim\|D \bar{v}\|_{L_{t}^{1}\left(\dot{B}_{p, 1}^{n / p}\right)}, \\
& \|\operatorname{Id}-A(t)\|_{\dot{B}_{p, 1}^{n / p}} \lesssim\|D \bar{v}\|_{L_{1}\left(0, t ; \dot{B}_{p, 1}^{n / p}\right)}, \\
& \left\|\partial_{t}(\operatorname{adj}(D X))(t)\right\|_{\dot{B}_{p, 1}^{n / p}} \lesssim\|D \bar{v}(t)\|_{\dot{B}_{p, 1}^{n / p}}, \\
& \left\|\partial_{t}(\operatorname{adj}(D X))(t)\right\|_{\dot{B}_{p, 1}^{n / p-1}} \lesssim\|D \bar{v}(t)\|_{\dot{B}_{p, 1}^{n / p-1}} \quad \text { if } p<2 n \\
& \left\|\operatorname{adj}(D X(t))^{T} A(t)-\operatorname{Id}\right\|_{\dot{B}_{p, 1}^{n / p}} \lesssim\|D \bar{v}\|_{L_{1}\left(0, t ; \dot{B}_{p, 1}^{n / p}\right.} .
\end{aligned}
$$

Proof: According to Lemma 2 and to (A.5), one may write

$$
\operatorname{Id}-\operatorname{adj}(D X(t))=\int_{0}^{t}(D \bar{v}-\operatorname{div} \bar{v} \operatorname{Id}) d \tau+P_{2}\left(\left(\int_{0}^{t} D \bar{v} d \tau\right)\right)
$$

where the coefficients of $P_{2}$ are at least quadratic polynomials of degree $n-1$. Given that $\dot{B}_{p, 1}^{n / p}$ is a Banach algebra and that (2.5) holds, we readily get the result. 
In order to prove the second estimate, we just use the fact that, under assumption (2.5), we have

$$
A(t)=(\operatorname{Id}+C(t))^{-1}=\sum_{k \in \mathbb{N}}(-1)^{k}(C(t))^{k} \quad \text { with } \quad C(t)=\int_{0}^{t} D \bar{v} d \tau,
$$

and that $\dot{B}_{p, 1}^{n / p}$ is a Banach algebra.

In order to prove the third inequality, we use the fact that, according to Lemma 2, we have

$$
\partial_{t}(\operatorname{adj}(D X))=\frac{\partial}{\partial t}\left(\operatorname{Id}+\int_{0}^{t}(\operatorname{div} \bar{v} \operatorname{Id}-D \bar{v}) d \tau+P_{2}\left(\int_{0}^{t} D \bar{v} d \tau\right)\right) .
$$

Hence

$$
\partial_{t}(\operatorname{adj}(D X))(t)=(\operatorname{div} \bar{v}(t) \operatorname{Id}-D \bar{v}(t))+d P_{2}\left(\int_{0}^{t} D \bar{v} d \tau\right) \cdot D \bar{v}(t)
$$

As the coefficients of $d P_{2}$ are polynomials of $n^{2}$ variables that vanish at 0 , we get

$$
\left\|\partial_{t}(\operatorname{adj}(D X))(t)\right\|_{\dot{B}_{p, 1}^{n / p}} \lesssim\|D \bar{v}(t)\|_{\dot{B}_{p, 1}^{n / p}}\left(1+\left\|\int_{0}^{t} D \bar{v} d \tau\right\|_{\dot{B}_{p, 1}^{n / p}}\right),
$$

hence (A.9). Proving (A.10) is similar: it is only a matter of using the continuity of the product from $\dot{B}_{p, 1}^{n / p-1} \times \dot{B}_{p, 1}^{n / p}$ to $\dot{B}_{p, 1}^{n / p-1}$, if $p<2 n$.

For proving the last inequality, we use the decomposition

$$
\operatorname{adj}(D X)^{T} A-\operatorname{Id}=(\operatorname{adj}(D X)-\mathrm{Id})^{T} A+{ }^{T}(A-\mathrm{Id}) .
$$

So combining Inequalities (A.7) and (A.8), and the fact that $\dot{B}_{p, 1}^{n / p}$ is a Banach algebra, we get the result.

Lemma 4. Let $\bar{v}_{1}$ and $\bar{v}_{2}$ be two vector-fields satisfying (2.5), and $\delta v:=\bar{v}_{2}-\bar{v}_{1}$. Then we have for all $p \in[1,+\infty)$,

$$
\begin{gathered}
\left\|A_{2}-A_{1}\right\|_{L_{\infty}\left(\mathbb{R}_{+} ; \dot{B}_{p, 1}^{n / p}\right)} \lesssim\|D \delta v\|_{L_{1}\left(\mathbb{R}_{+} ; \dot{B}_{p, 1}^{n / p}\right)}, \\
\left\|\operatorname{adj}\left(D X_{2}\right)-\operatorname{adj}\left(D X_{1}\right)\right\|_{L_{\infty}\left(\mathbb{R}_{+} ; \dot{B}_{p, 1}^{n / p}\right)} \lesssim\|D \delta v\|_{L_{1}\left(\mathbb{R}_{+} ; \dot{B}_{p, 1}^{n / p}\right)}, \\
\left\|\partial_{t}\left(\operatorname{adj}\left(D X_{2}\right)-\operatorname{adj}\left(D X_{1}\right)\right)\right\|_{L_{1}\left(\mathbb{R}_{+} ; \dot{B}_{p, 1}^{n / p}\right)} \lesssim\|D \delta v\|_{L_{1}\left(\mathbb{R}_{+} ; \dot{B}_{p, 1}^{n / p}\right)}, \\
\left\|\partial_{t}\left(\operatorname{adj}\left(D X_{2}\right)-\operatorname{adj}\left(D X_{1}\right)\right)\right\|_{L_{2}\left(\mathbb{R}_{+} ; \dot{B}_{p, 1}^{n / p-1}\right)} \lesssim\|D \delta v\|_{L_{2}\left(\mathbb{R}_{+} ; \dot{B}_{p, 1}^{n / p-1}\right)} \quad \text { if } p<2 n .
\end{gathered}
$$

Proof: In order to prove the first inequality, we use the fact that, for $i=1,2$, we have

$$
A_{i}=\left(\mathrm{Id}+C_{i}\right)^{-1}=\sum_{k \geq 0}(-1)^{k} C_{i}^{k} \quad \text { with } \quad C_{i}(t)=\int_{0}^{t} D \bar{v}_{i} d \tau .
$$

Hence

$$
A_{2}-A_{1}=\sum_{k \geq 1}\left(C_{2}^{k}-C_{1}^{k}\right)=\left(\int_{0}^{t} D \delta v d \tau\right) \sum_{k \geq 1} \sum_{j=0}^{k-1} C_{1}^{j} C_{2}^{k-1-j} .
$$

So using the fact that $\dot{B}_{p, 1}^{n / p}$ is a Banach algebra, it is easy to conclude to (A.13). 
The second inequality is a consequence of Lemma 2 and of the Taylor formula which ensures that, denoting $\delta C:=C_{2}-C_{1}$,

$$
\operatorname{adj}\left(D X_{2}\right)-\operatorname{adj}\left(D X_{1}\right)=(\operatorname{Tr}(\delta C)) \operatorname{Id}-\delta C+d P_{2}\left(C_{1}\right)(\delta C)+\frac{1}{2} d^{2} P_{2}\left(C_{1}, C_{1}\right)(\delta C, \delta C)+\cdots
$$

where the coefficients of $P_{2}$ are polynomials of degree $n-1$. As the sum is finite and $\dot{B}_{p, 1}^{n / p}$ is a Banach algebra, we get (A.14).

In order to prove the last two estimates, it is only a matter of differentiating the above relation with respect to $t$. Keeping in mind the definition of $C_{1}$ and $C_{2}$, we get

$$
\partial_{t}\left(\operatorname{adj}\left(D X_{2}\right)-\operatorname{adj}\left(D X_{1}\right)\right)=(\operatorname{div} \delta v) \operatorname{Id}-D \delta v+d P_{2}\left(C_{1}\right) \cdot \partial_{t} \delta C+d^{2} P_{2}\left(C_{1}\right)\left(\partial_{t} C_{1}, \delta C\right)+\cdots .
$$

Then using the product laws in Besov spaces yields the desired inequalities.

Finally, we have to justify that the multiplier space $\mathcal{M}\left(\dot{B}_{p, 1}^{n / p-1}\right)$ contains characteristic functions of $C^{1}$ bounded domains, if $p>n-1$. This is a consequence of the following lemma.

Lemma 5. Let $\Omega$ be the half-space $\mathbb{R}_{+}^{n}$ or a bounded domain of $\mathbb{R}^{n}$ with $C^{1}$ boundary. Assume that $s \in \mathbb{R}$ and $p, q \in[1, \infty]$ are such that

$$
-1+\frac{1}{p}<s<\frac{1}{p}
$$

Then the characteristic function $\chi_{\Omega}$ of $\Omega$ belongs to the space $\mathcal{M}\left(\dot{B}_{p, q}^{s}\left(\mathbb{R}^{n}\right)\right)$.

Proof: This result which belongs to the mathematical folklore is closely related to the fact that under Condition (A.17), functions in $\dot{B}_{p, q}^{s}(\Omega)$ extended by 0 on the whole space, belong to $\dot{B}_{p, q}^{s}\left(\mathbb{R}^{n}\right)$. In the case where $\Omega$ is the half-space $\mathbb{R}_{+}^{n}$ the lemma has been proved in [7], Prop. 3 .

If $\Omega$ is a bounded $C^{1}$ domain, then one may find a finite number $N$ of $\mathcal{C}_{c}^{\infty}\left(\mathbb{R}^{n}\right)$ functions $\phi_{i}$ and $C^{1}$ diffeomorphisms $\psi_{i}$ so that for any $u \in \dot{B}_{p, q}^{s}\left(\mathbb{R}^{n}\right)$,

$$
u 1_{\Omega}=\sum_{i=1}^{N} u \phi_{i} 1_{\Omega} \quad \text { and } \quad\left(u \phi_{i} 1_{\Omega}\right) \circ \psi_{i}=1_{\mathbb{R}^{+}} \cdot\left(\left(u \phi_{i}\right) \circ \psi_{i}\right) .
$$

Now, because Condition (A.17) is satisfied, the space $\dot{B}_{p, q}^{s}\left(\mathbb{R}^{n}\right)$ is stable by multiplication by smooth compactly supported functions, and by $C^{1}$ change of variables (see e.g. [8], Chap. 2). Therefore the functions $\left(u \phi_{i}\right) \circ \psi_{i}$ belong to $\dot{B}_{p, q}^{s}\left(\mathbb{R}^{n}\right)$, too. Using again the stability of this space by multiplication by $1_{\mathbb{R}^{+}}$, one may thus conclude that $\left(u \phi_{i} 1_{\Omega}\right) \circ \psi_{i} \in \dot{B}_{p, q}^{s}\left(\mathbb{R}^{n}\right)$. Hence $u \phi_{i} 1_{\Omega}$ is in $\dot{B}_{p, q}^{s}\left(\mathbb{R}^{n}\right)$ for all $i \in\{1, \cdots, N\}$. This completes the proof of the lemma.

Acknowledgment. The second author (PBM) has been partly supported by Polish MN grant No. N N201 547438 and by Foundation for Polish Science in fr. EU European Regional Development Funds (OPIE 20072013). He thanks the University Paris-Est Créteil, where a part of the paper has been performed, for its kind hospitality.

\section{REFERENCES}

[1] H. Abidi: Équation de Navier-Stokes avec densité et viscosité variables dans l'espace critique, Rev. Mat. Iberoam., 23(2), pages 537-586 (2007).

[2] H. Abidi and M. Paicu: Existence globale pour un fluide inhomogène, Annales de l'Institut Fourier, 57(3), pages $883-917$ (2007).

[3] S. Antontsev, A. Kazhikhov and V. Monakhov: Boundary value problems in mechanics of nonhomogeneous fluids. Studies in Mathematics and its Applications, 22. North-Holland Publishing Co., Amsterdam, 1990.

[4] H. Bahouri, J.-Y. Chemin and R. Danchin: Fourier Analysis and Nonlinear Partial Differential Equations, Grundlehren der mathematischen Wissenschaften, 343, Springer (2011).

[5] H.J. Choe and H. Kim: Strong solutions of the Navier-Stokes equations for nonhomogeneous incompressible fluids, Comm. Partial Differential Equations, 28 no. 5-6, 1183-1201 (2003). 
[6] R. Danchin: Density-dependent incompressible viscous fluids in critical spaces, Proceedings of the Royal Society of Edinburgh, Sect. A, 133(6), pages 1311-1334 (2003).

[7] R. Danchin and P. B. Mucha: A critical functional framework for the inhomogeneous Navier-Stokes equations in the half-space, J. Funct. Anal., 256(3), pages 881-927 (2009).

[8] R. Danchin and P.B. Mucha: Critical functional framework and maximal regularity in action on systems of incompressible flows, in progress.

[9] P. Germain: Strong solutions and weak-strong uniqueness for the nonhomogeneous Navier-Stokes equation, J. Anal. Math., 105, pages 169-196 (2008).

[10] D. Hoff: Uniqueness of weak solutions of the Navier-Stokes equations of multidimensional compressible flow, SIAM Journal on Mathematical Analysis, 37(6), pages 1742-1760 (2006).

[11] O. Ladyzhenskaya and V. Solonnikov: The unique solvability of an initial-boundary value problem for viscous incompressible inhomogeneous fluids, Journal of Soviet Mathematics, 9, pages 697-749 (1978).

[12] P.-L. Lions: Mathematical Topics in Fluid Dynamics, Vol. 1 Incompressible Models, Oxford University Press (1996).

[13] V. Maz'ya and T. Shaposhnikova: Theory of Sobolev multipliers. With applications to differential and integral operators. Grundlehren der Mathematischen Wissenschaften, 337, Springer (2009).

[14] P.B. Mucha: On weak solutions to the Stefan problem with Gibbs-Thomson correction, Differential and Integral Equations, 20(7), 769-792 (2007).

[15] P.B. Mucha and W.M. Zajączkowski: On local existence of solutions of free boundary problem for incompressible viscous self-gravitating fluid motion, Applicationes Mathematicae, 27(3), pages 319-333 (2000).

[16] V.A. Solonnikov: On the nonstationary motion of isolated value of viscous incompressible fluid, Izv. $A N$ SSSR, 51(5), pages 1065-1087 (1987).

(R. Danchin) Université Paris-Est, LAMA, UMR 8050, 61 avenue du GÉnéral de Gaulle, 94010 Créteil Cedex, France.

E-mail address: danchin@univ-paris12.fr

(P.B. Mucha) Instytut Matematyki Stosowanej i Mechaniki, Uniwersytet Warszawski, Ul. BaNACHA 2, 02-097 WARszaWA, Poland.

E-mail address: p.mucha@mimuw.edu.pl 